adults; the non-specific tuberculosis only causes an ordinary inflammatory reaction in the tissues, but is capable of being the "initial disease"; at first hidden, it only expresses itself at the peripheries by specific lesions of ordinary appearance, in the first instance; throwing off the mask more or less suddenly, it finally takes the classical appearance of granular and excavating lesions.

In short, it is important to remember that rheumatism can show itself locally with all the alarming aspects of a true mastoiditis, with such a symptomatology that it may lead to too hastyintervention.

Everything being considered, there are only present in the occurrence the subcutaneous rheumatismal cdemas of the mastoid region, transitory odemas occasionally parallel to deep-seated auricular tronbles, the analysis of which would aroid useless mastoid operation. These local and subcutaneous affections are liable to occur in these transitional arthritic subjects who, having sprung from decidedly and thoroughly arthritic parents, have exhausted their power of resistance and become progressively the types of a particular constitution. These types are-according to Poucet's expression- "paratuberculous" species of the degenerated arthritics, yet refractory to the severe internal tuberculoses; they are very favourable, on the contrary, to local tuberculoses in the skin, joints or elsewhere, spoken of as " petites tuberculoses," with which they slowly exhaust themselves.

\title{
THE MODERN TREATMENT OF SYPHILIS, ESPECIALLY IN REGARD TO THE UPPER RESPIRATORY PASSAGES. ${ }^{1}$
}

\author{
By W. A. Lieven, M.D. \\ (Aix-la-Chapelle).
}

Wiтнix the last few years considerable progress has been made in the diagnosis of syphilis. The microbe of syphilis, the Spirochata pallida, has been found, and we now know from the investigations of Wassermann, Bruck, and Neisser that the serum of a syphilitic contains bodies which are only to be found in people infected with syphilis. On the other hand we have not progressed very far as regards the means of fighting against this disease. Mercury and iodide are still the most important, if not the only reliable, remedies. The experiments in serum-therapy have un-

${ }^{1}$ Introduction to a Discussion in the Laryngological Section of the Royal Society of Medicine, on January 8, 1909. 
fortunately all failed, and the preparations of arsenic which have been employed during the last two years are neither reliable in their results nor are we acquainted with the exact indications for their use. I will speak first of the various anti-syphilitic drugs, and in the second place give an outline of the methods of their employment.

Mercury still remains the most important remedy. Neisser in Batavia studied the influence of all the different drugs which are employed in the treatment of syphilis upon a large number of anthropoid apes and monkeys, and the results showed (1) that mercury not only assists the organism to defend itself against the spirochrta, but also kills the microbe; (2) that inoculations made from the internal organs of a syphilitic monkey which has been sufficiently treated with mercury are incapable of transferring the virus to another animal; (3) that a syphilitic monkey which has been brought sufficiently under the influence of mercury to effect a cure can be reinfected a second time with syphilis. I should also like to remark that similar results can be obtained by the use of atoxyl. Unfortunately the human race cannot tolerate such large doses of atoxyl without danger to the optic nerve. All these facts afford theoretical confirmation of the experiences of daily practice.

There are different methods of administering mercury to the organism. When given by the mouth absorption is carried out by the gastro-intestinal tract, when injected, by the subcutaneous or muscular tissue, whilst in the case of inunction the skin and the lungs participate in the absorption of the metal into the system.

As regards the treatment of syphilis by the mouth, in Germany we are all agreed that this method is insufficient to obtain a satisfactory result. I have not only seen a great many relapses of a secondary and early tertiary character, occurring during the first two years of the course of the disease whilst mercury was constantly being taken, but during seventeen years' practice in Aix-la-Chapelle I have noticed that after no other form of treatment were there so many severe symptoms in connection with the central nervous system as after a pill cure, as it is still carried out in England. In addition to its inefficiency in combating the disease it has the great drawback of very easily producing a chronic enteritis, and when taken for any length of time a chronic stomatitis of a most distressing character. The stomatitis, due to rubbings or injections, is generally shown by a dark red mucous membrane, whilst the gums, during treatment by the mouth, often present a pale and 
dirty appearance, and exhibit a tendency to considerable retraction. At the same time this form of stomatitis is not by any means a sign that there is an energetic action upon the syphilis, because it is not a rare occurrence to find recent mucous patches on the mucosa of a patient who is suffering from this particular rariety of stomatitis. The theoretical investigations made by Bürgi and others furnish an explanation for this. He found that the mercury is rapidly eliminated and disappears from the urine within a few days after the administration of it is stopped.

The second method of treatment of which we must speak is that of hypodermic injections. We inject either soluble preparations of mercury or insoluble ones suspended in an oily medinm. 'The soluble ones were introduced by Lewin, of Berlin, in 1867. His custom was to inject a 1 per cent. solution of corrosive snblimate. Since then a great many different preparations have been employed which it is impossible to describe here. I am in the habit of using the French "Bi-iodure de Mercure" ( 2 per cent. solution), because I find it practically painless and its effect as good as any of the others. An injection of 1 c.c. or $15 \mathrm{~m}$ is given daily for twenty to thirty days. There are two drawbacks to the administration of soluble salts of mercury: (1) The necessity for seeing the doctor every day, and (2) the fact that the mercury, though effective at the time, very soon disappears from the system. Relapses occur more frequently than aftcr the insoluble preparations.

Of the latter the following are in general use: Calomel, grey oil and salicylate of mercury. The two first are injected in a 40 per cent. strength. One employs a special syringe (Barthélémy's), and in the case of calomel injects up to eight divisions once a week, or if huile grise is used up to twelve divisions once a weck. Ont division of this syringe is masured to contain one centigramme of mercury of a 40 per cent. preparation. The syringe is indispensable for calomel and grey oil, as it is impossible to give exact duseswith the usual Pravaz syringe. Six to eight injections of calomel, ten to twelve of grey oil, and eight to ten full Pravaz syringes (1 c.e. or $15 \mathrm{~m}$ ) of salicylate, are considered sufficient for a full coursec. There is no donbt that these injections have a great many advantages. We are certain of the quantity the patient is getting, and the treatment can be carried out without giving rise to any suspicion in the patient's surroundings. The method also is convenient, as the patient requires to see his doctor only once or twice a week. Last, but not least, there is the fact that the results of this kind of treatment are very satisfactory. 
The most effective of the three preparations is calomel, whilst the grey oil has a much slower, but generally sufficient, effect. Between these two stands the salicylate of mercury, which it is right to say is used with excellent results by many physicians in Germany. Its curative effect is sufficient, it is generally painless, and there seems to be hardly any danger in its administration.

This leads me to speak of the disadvantages of the insoluble preparations. Calomel is extremely painful, although this drawback has to a certain extent been overcome in Levy-Bings and Neisser's new suspensions. Aseptic abscesses are common, and one sometimes observes that two or three days after such an injection the patient has an attack of shivering followed by some fever. This attack is called by the French "la grippe mercurielle." Against these injections is to be urged the fact that when once an injection is made the medical man is no longer in a position to influence the degree of rapidity of the absorption. When this takes place too rapidly severe symptoms may appear. It is possible that a dose of an insoluble salt of mercury may lie dormant for months, and then pass rapidly into the circulation, giving rise to urgent symptoms. Even fatal results have occurred from this canse. The salicylate seems to be practically without danger, and I therefore consider it the only preparation fit for the routine treatment of syphilis, whilst I reserve calomel for the malignant forms, in which a rapid result is requisite, and everything else has failed.

Whichever insoluble salt we employ, it will always be most improtant that the "technique" should be carried out most carefully. First we should introduce the needle, in order to ascertain whether a vein has been penetrated. For the same reason I always inject into the upper part of the buttock, above a horizontal line joining the tops of the trochanter major, and preferably in the upper and onter quadrant. Under no circumstances whatever should injectims be given in cases of diabetes or Bright's disease.

In our country, owing to the many disadvantages, if not dangers, associated with injections, the majority of the profession mefer treatment by inunction. The teachings of sigmund have made it clear that inunctions can be carried ont without necessarily producing mercurialism. The evolution of the present method of innuction is due to these principles. At Aix-la-Chapelle the routine of rubbings has especially been brought to perfection, and it is due to that place that this treatment has regained its reputation on the Continent of being the method which combines the greatest therapentical effect with no danger whatsoever to the patient, because 
one can at any time stop the absorption of mercury by simply washing off the ointment. I admit that the treatment by inunctions is difficnlt to conceal from the patient's surroundings, and that the cure deserves the name of being dirty and disagreeable, but in spite of this its advantages are so great that whenever I have asked a medical man in onr country what kind of treatment he would prescribe for himself, there was no one who did not reply, "Inumction."

Inunction, however, ought to be carried out very carefully. The process is as follows: With the bare hand the requisite quantity-r.g. 4 grm. to 5 grm. of 33 per cent. grey ointment-is evenly rubbed into the skin by the patient himself for a period of at least twenty minutes, and after this time, if the innction has been properly carried out, the skin should appear dry and not shiny. On the first day both calves are rubbed; on the second, the left; on the third day, the right thigh; on the fourth day, the abdomen and flanks; on the fifth day, both arms. During these five days no bath is taken, and there should be no change of underwear. On the sixth day the whole body is thoroughly washed with soap in a bath. These courses of five days are repeated again and again until the requisite number of rubbings has been reached. If there is a reliable massemr at hand, both thighs should be rubbed on the second day, and the back on the fifth day. This is the best way of carrying out the cure at home or at the hospital.

At Aix, however, we enable the patient, by the use of the thermal waters, to absorb and to tolerate much larger quantities of mercurial ointment than those mentioned above. The daily rubbing is preceded by a sulphur bath of about $95^{\circ} \mathrm{F}$., in which the part which is to be rubbed later is carefully washed with soap. The alkaline water softens and removes the superficial layers of the epidermis, thus opening the pores for the absorption of the ointment. 'The temperature of the bath is not high enough to remove the ointment in the bath unless it is washed off with soap. The mercury does not penetrate the normal skin, but is rubbed into the pores, where it is converted by the secretion of the sebaceous and the sweat-glands into combinations capable of being absorbed by the system, and when absorbed circulates in the body as an albuminate. It is also absorbed by inhalation. 'The baths and drinking waters promote metabolism, thus giving the mercury an opportunity to enter into new combinations with fresh albumen, and consequently to be distributed to its fullest extent throughout the system.

The two most unpleasant symptoms of mercurialism-namely, stomatitis and colitis-may be observed with every form of 
treatment, but they can be almost certainly prevented by careful attention being bestowed on the buccal cavity and on the regulation of the bowels. During the cure the teeth must be cleansed after each meal by means of a soft tooth-brush with a tooth-paste of salol and chlorate of potash. 'The mouth also must be rinsed every hour with a solution of aluminium acetico-tartaricum. Mercurial ulcerations of the guns are cured in a few days by being touched with a concentrated solution of chromic acid. It is essential to remove all débris from the space between the gums and teeth before painting. Naturally, careful rinsing of the mouth after the application of so strong a drug is essential.

The intestinal tract should be attended to, and care should be taken that the bowels are thoroughly opened every day in order to eliminate the mercury secreted into the intestines. 'The sulphur water at $\mathrm{Aix}$ is often sufficient to ensure a daily motion, and the hydrogen sulphide in it acts upon the mercury so as to produce the comparatively imnocuous black sulphide, thus reducing the irritation of the gastro-intestinal mucosa to a minimum.

In cases where diarrhoa occurs, even if associated with blood in the stools, a dose of 25 drops of laudanum, if necessary repeated again in eight to ten hours, and the removal of the ointment by neans of a soft bath, will certainly stop the diarrhœa.

The second remedy-namely, iodide-is more useful in tertiary manifestations. Its chief action consists in promoting absorption of specific neoplasms which characterise this stage. One, however, obtains good results with iodide in certain secondary symptoms, such as vegetating patches, which are chiefly found at the entrance of the nasal passages and on the floor of the mouth. The preparation which works most quickly is iodide of potassium, and no other preparation of iodine equals it in effect, so that it should invariably be used in cases of imminent danger to life or important functions, or in those cases in which a rapid diagnosis is required, as, for instance, in the case of a growth of doubtful character. Unfortunately, very often this drug or other preparations of iodine camnot be given on account of the occurrence of severe iodism. In these cases a daily prescription of $15 \mathrm{gr}$. of sulphanilic acid in $7 \mathrm{oz}$. of water will be found most successful in preventing this uupleasant symptom.

If the patient should be quite unable to take iodide of potash by the mouth, I prescribe sajodine, 3 to 8 tablets of $\frac{1}{2} \mathrm{grm}$. per day. It has a mild and prolonged effect, and is eliminated more slowly than iodide of potash. 'This is still more the case with 
subcutaneous injections of iodipin, which I inject in doses of $20 \mathrm{grm}$. to $30 \mathrm{grm}$. three times a week. The total course is $250 \mathrm{grm}$. This quantity is sufficient to put the system under the continuous mild effect of iodine for about six months. This method, therefore, is of great prophylactic value, and very useful when the patient cannot be trusted to take iodide by the mouth as regularly as he ought.

As you all know preparations of arsenic have recently been tried in the treatment of syphilis. The sodium amylarsenate (atoxyl) has been given up in our country on account of the repeated occurrence of umpleasant symptoms; several cases may be mentioned in which total atrophy of the optic nerve resulted. Researches undertaken with the object of finding another less poisonous preparation of arsenic have led in England to experiments with soamin (sodium para-aminophenylarsenate), and in Germany to experiments with arsacetin (acetylanilarsenate), which is similar to the orsudan of Burroughs and Wellcome.

The number of cases treated with these two preparations is not sufficient to induce me to recommend the use of these drugs in general practice. I think Neisser is right when he says that only perhaps after twenty years of experience with an enormous number of cases shall we be entitled to say anything about the real therapeutic value of arsenic.

After this scheme of general treatment I should now like to speak about the time when treatment should be commenced, and the duration of the course requisite for a complete cure. I am of opinion that in the case of a sore which is suspected to be of a primary nature the diagnosis ought to be certain before treatment is commenced. This is especially necessary, because chancres of the upper respiratory tract are sometimes extremely difficult to diagnose. If the spirochreta cannot be found one must wait for a few weeks for the appearance of the roseola or examine the case by the serum test, which, however, is unfortunately only to be obtained several weeks after the appearance of the chancre. An exception should be made in those cases where the doubtful sore is in a prominent position, or where the patient comes into close contact with other people.

For the first course I generally prescribe forty to fifty rubbings of $5 \mathrm{grm}$. each. At Aix, if the patient stands mercury ivell, I let him have two rubbings jer day of 4 grm. to 5 grm. each during the last fortnight. If he is anamic I give him three to four injections of sodium arsenate three to four times a week. Though 
the primary sore and secondary eruptions generally disappear within a fortnight after commencement of the cure, it ought always to be carried out to its full extent. The care of the mouth should be continued for a fortnight after each course. Three further courses of rubbings should follow at intervals of six months. If there has not been a relapse during the first year of treatment the fourth course may be postponed until the end of the second year. I wish to lay stress upon the necessity of carrying out these treatments, even if a relapse should not have occurred.

Should it be impossible to give inunctions on account of social reasons or owing to the irritability of the skin, I substitute eight to ten injections of salicylate of mercury in vasenol. When rubbings cannot be given and intra-muscular injections are not tolerated, then and then only do I decide to give internal treatment. I am in the habit of prescribing the tannate of mercury in doses of 1 decigramme three times a day in pills. These are taken for six weeks, followed by an interval of two months' rest, and so on for a period of two years.

Before speaking about the local treatment of the different forms of syphilis in the nose, mouth and throat, I should like to say that in most cases general treatment suffices, and local methods can be lispensed with. If the chancre is situated on the lips or on the outside of the nose it ought, for cosmetic and prophylactic reasons, to be covered by a mercury plaster (Beyersdorff No. 15). For chancre inside the nose an indifferent spray, followed by the insufflation of nosophen, is sufficient in every case. Chancres in the mouth or pharynx ought not to be cauterised, as they heal very quickly under general treatment, but they may early require the dusting on of some orthoform, as they are extremely painful.

Relapses of a secondary type on the mucons membranes are generally more resistant to treatment than lesions occurring at the commencement of the disease, which as a rule promptly yield to general treatment. The most useful remedy for patches of the erosif or "ulcerated" type (Fournier) is the application of a concentrated solution of chromic acid; the mucosa ought to be carefully dried before the drug is administered in order to prevent it from spreading. If a very adhesive scab is required one shonld paint over the chromic acid a 10 per cent. solution of silver nitrate. The scab will then become bright red in colour, owing to the formation of silver chromate. Some patients with ulcerated patches on the edges of the tongue suffer considerably from profuse salivation, which easily loosens the scabs produced by 
cauterisation. The giving of belladonna will in many cases minimise this. Mucous plaques of the introitus narium or cracks at the angles of the mouth should, after cauterisation, be covered with 5 per cent. white precipitate ointment.

If shortly after an energetic course of treatment a relapse occurs with secondary symptoms on the mucosa, it is not wise to at once give another full course of treatment. It is often possible to overcome these manifestations simply by local treatment. Should this not be followed by success another mild treatment may be advisable, such as wearing a mercolint flannel on the chest or taking mercury tannate pills for three to seven weeks. By these measures it is practically always possible to keep the patient going until the time arrives for beginning the next full course of rubbings, as specified in our scheme. Smoking is very often the reason of the constant relapses, and you will often find that from the moment the patient abstains from tobacco the patches cease to appear.

In any case one should try to keep as near as possible to the chief courses of treatment as prescribed in the scheme. It stands to reason that extremely severe cases may make it necessary to give the patient more frequent treatments. At the end of the first year my patients begin to take iodide. I give $10 \mathrm{gr}$. three times daily for three to four weeks, after the third and fourth cure. Or I give a course of injections of iodipin up to a total of $250 \mathrm{grm}$. during the inunction treatment itself.

The laryngologist does not often have an opportunity of observing a case of syphilis from the beginning. He sees more cases of a tertiary nature, where iodide is the sovereign remedy for a quick result. 'Thirty grains daily ought to be given at least. If one of the substitutes of $\mathrm{KI}$ is to be given one must bear in mind that a favourable result cannot be expected so quickly. In ulcerated tertiaries I always give iodide of potash alone for four or five days, and only when, from its appearance, the ulcer shows a tendency to healing, do I start inunctions. After the tertiary ulcers are healed indide is stopped, and iodipin injections are employed whilst the rubbings are continued. We expect the inunctions and iodipin together, on account of their prolonged effect, to prevent relapses, which iodide of potash alone often fails to do.

In addition to tertiary cases of a regular type, there are others that from the very beginning show a malignant character. They sometimes show themselves a few months after the primary sore; sometimes they even develop from persistent secondary eruptions. The malignant form of syphilis is often not cured by ordinary 
courses of mercury and iodide, but gets worse and worse. I have seen cases of ulcerations in the upper air-passages which after each dose of iodide showed a sudden breaking down of the infiltrated tissue, and when the iodide was stopped the reaction ceased.

In these desperate cases it is advisable to try injections of calomel, which should not be given at the same time as the iodide. After a few injections of calomel I have seen a marvellous improvement take place, and this was followed by the extraordinary fact that afterwards these cases could be treated in a similar manner to ordinary tertiary cases.

As local treatment I recommend mercurial plaster for all ulcers of the lip and the outside of the nose; for those in the nose itself tampons, covered with a 10 per cent. ointment of europhen, have a cleansing and healing effect. The crusts are also easily removed by these tampons. If the granulations of tertiary sores are not sufficient, they ought to be painted with tincture of iodine. This is often required on the posterior wall of the pharynx, as there is very little tendency to healing owing to the very poor vascularisation of this part. Sequestra ought not to be dealt with until they become loose. It may be necessary to cut them into several pieces by nasal forceps if they are too large to be pulled out in one piece. In two cases I made an incision in the naso-labial fold in order to remove a large piece of bone. The extraction, even of large sequestra, is generally comparatively easy, because the septum is, as a rule, partially destroyed before the other parts of the nose become involved, and thus more room is afforded for handling the necrosed bone. I will not refer here either to the treatment of perforations of the hard and soft palate or to the plastic operations or paraffin injections which are employed in the treatment of deformities of the nose.

Adhesions of the soft palate to the posterior wall of the pharynx ought to be separated if there is enough of the muscular tissue left to ensure the closure of the naso-pharynx during deglutition. If the patient is unable to do this after the operation he is worse off than before, for his voice retains an abnormal character, and in addition to this all food passes into the naso-pharynx and the nose. Several forms of apparatus have been introduced to keep the soft palate separated from the posterior wall of the pharynx after operation: a vulcanite plate fixed by a spring to the teeth, or an indiarubber ball lying in the naso-pharynx, which is filled with air by means of a tube passing through the nose. A case exhibiting a good result was shown at this Society, the 
apparatus used being a lead plate fixed by strings which passed through the nose and mouth.

Tertiary processes in the larynx require energetic general treatment, as there is a marked tendency to cicatricial narrowing of the larynx in these cases. We must bear in mind that fibroid metamorphosis may result from infiltration of the larynx without any sign of ulceration. The methods of dilating these contractions are the same as those employed in the treatment of narrowing of the larynx from other causes.

I wish to draw your attention to the rare tertiary cases, first described by Sir Felix Semon, in which warty excrescences appear in the larynx, and if localised on the vocal cords may canse considerable dyspnœa. These new formations ought not to be treated by local measures or by operation, as they always yield to general treatment, though I do not wish to hide the fact that an energetic and prolonged treatment is necessary.

Last, but not least, I should like to draw your attention to the necessity of treating the swellings of the local glands, which nearly always persist to a certain degree after the general treatment. I advise the patient to massage these swellings with $\frac{1}{2} \mathrm{grm}$. of blue ointment before going to bed for a period of a fortnight. This should be repeated from time to time.

\section{SOCIETIES' PROCEEDINGS.}

\section{PROCEEDINGS OF THE ROYAL SOCIETY OF MEDICINE-LARYNGOLOGICAL SECTION.}

Meeting on February 5, 1909.

Dr. Dundas Grant, President, in the Chair. Abstract of Proceedings by Dr. Dan MoKenzie.

The following cases and specimens were shown:

Case of Tertiany Specific Complete Atresia of the Posterior Nares in a Middle-Aged Woman.

By Dr. Dundas Grant.

Operation, December 18, for detachment of the soft palate from the posterior wall of the pharynx, including removal of a 